\title{
ELECTRICAL CONTACTS TO EVAPORATED CADMIUM SELENIDE
}

\author{
G. DE MEY \\ Laboratory of Electronics, Ghent State University, Belgium
}

(Received June 17, 1973; in final form November 18, 1973)

\begin{abstract}
The voltage-current characteristics of evaporated gold-CdSe contacts have been experimentally investigated. In contrast to the experiments of Weimer with Cadmium Sulphide, it was found that CdSe makes no ohmic contact with the underlying gold layers. The reverse biased contact has a zener-like behaviour. With silver ink contacts deposited upon the CdSe, perfectly ohmic contacts were obtained.
\end{abstract}

Firstly the well known theory of a metal n-type semiconductor contact is summarised. The metal and the semiconductor are characterised by their work functions $\phi_{\mathrm{M}}$ and $\phi_{\mathrm{S}}$ respectively. For a given semiconductor the energy difference between the zero energy level $\mathrm{E}_{0}$ outside the semiconductor, and the bottom of the conduction band at the surface is a fixed quantity, the electron affinity $\chi$. When the doping concentration is known, the energy difference $\mathrm{E}_{\mathrm{cb}}-\mathrm{E}_{\mathrm{F}}$ between the bottom of the conduction band and the Fermi level in the bulk can be calculated. The work function $\phi_{\mathrm{S}}$ is then:

$$
\phi_{\mathrm{S}}=\chi+\mathrm{E}_{\mathrm{cb}}-\mathrm{E}_{\mathrm{F}}
$$

Initially the case $\phi_{\mathrm{M}}>\phi_{\mathrm{S}}$ is considered. Figure la shows the situation before and after the contact was made. The metal will be negatively charged throughout a shallow surface layer. In the semiconductor a depletion layer is built up. So a diode behaviour of the junction is to be expected. In the other case $\phi_{\mathrm{M}}$. $<\phi_{\mathrm{S}}$ (fig. 1b) an accumulation layer will be formed in the semiconductor. This junction shows a non rectifying contact. One says that the junction is ohmic. However, in the case that $\phi_{\mathrm{M}}>\phi_{\mathrm{S}}$ an ohmic junction can be formed when the carrier concentration is very high. In this case the thickness of the depletion layer is so small that conduction takes place by quantum mechanical tunneling through the space charge layer ${ }^{1}$.

Weimer ${ }^{2,3}$ has found that gold evaporated on $\mathrm{CdS}$ gives no ohmic contact, while $\mathrm{CdS}$ evaporated upon gold makes a good ohmic contact. His explanation is, that the first condensed semiconductor layer was $\mathrm{Cd}$ rich, and thus heavily doped with n-type charge carriers. The space charge layer becomes very thin and conduction takes place by quantum mechanical tunneling.

In the experiment $\mathrm{CdSe}$ was used as semiconducting material and gold for the metallic contacts. The materials were evaporated from a heated tungsten boat in a vacuum of about $10^{-6}$ Torr. A $1^{\prime \prime}$ square corning glass was used as substrate. Before the deposition of the semiconductor, gold contacts were evaporated on the cold substrate. The geometry used was a cross shaped Hall generator (Figure 2) with $\mathrm{h}=8 \mathrm{~mm}$ and $1=4 \mathrm{~mm}^{4}$. The thicknesses of the semiconductor and the gold layer were $1000 \AA$ and $500 \AA$ respectively, and the area of the overlapping region was $2 \mathrm{~mm}^{2}$ in order to assure good contact. The geome try offers the advantage that Hall mobility and carrier concentration on the one hand and I-V characteristics on the other hand can be measured on the same semiconductor sample. If the hypothesis of Weimer were also true for CdSe, the contacts should be ohmic. However the contrary is found. Figure 3 shows the I-V characteristics measured between two opposite contacts for various current ranges. The current was supplied by the Fluke programmable current calibrator, model 3330B. This current source provides a stable current even in the nanoampere range. The potential between the contacts was measured with the Fluke high impedance voltmeter/ null detector model $845 \mathrm{~A}$. Because the internal impedance of the voltmeter is $10 \mathrm{M} \Omega$, some numerical corrections were necessary to find the true current through the sample. For low voltages the sample looks like a resistor of $11 \mathrm{M} \Omega$. For high values of the voltage the curve has a slope of $113 \mathrm{k} \Omega$. When the current goes from the semiconductor towards the metal, this contact is a reverse biased metal- 


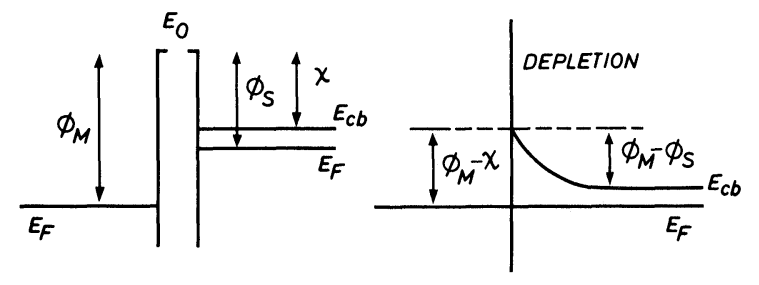

NO CONTACT
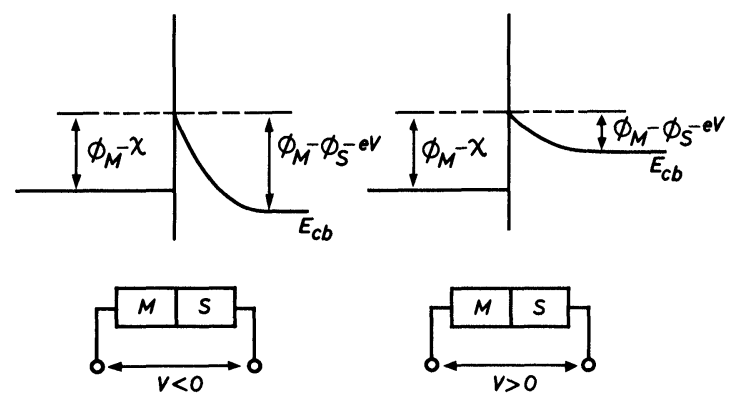

REVERSE BIAS

FORWARD BIAS
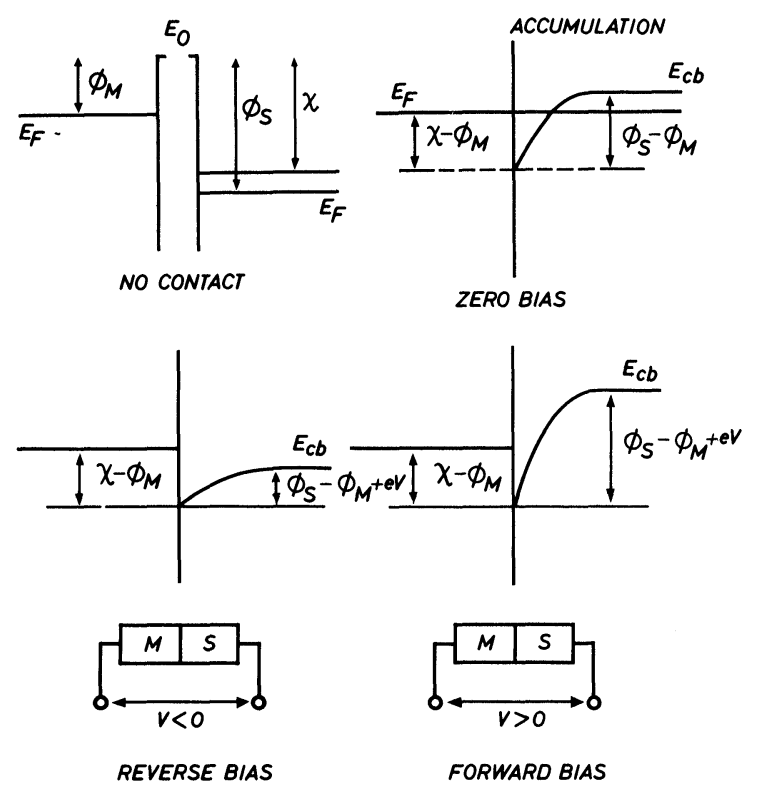

FIGURE 1 Energy bands at the metal-semiconductor interface.

semiconductor diode. The semiconductor can be assimilated to a $113 \mathrm{k} \Omega$ resistor. Supposing that there is negligible voltage drop over the forward biased diode one can construct the reverse characteristic of an Au-CdSe diode. This result is shown in figure 4. This characteristic is very similar to the voltage-

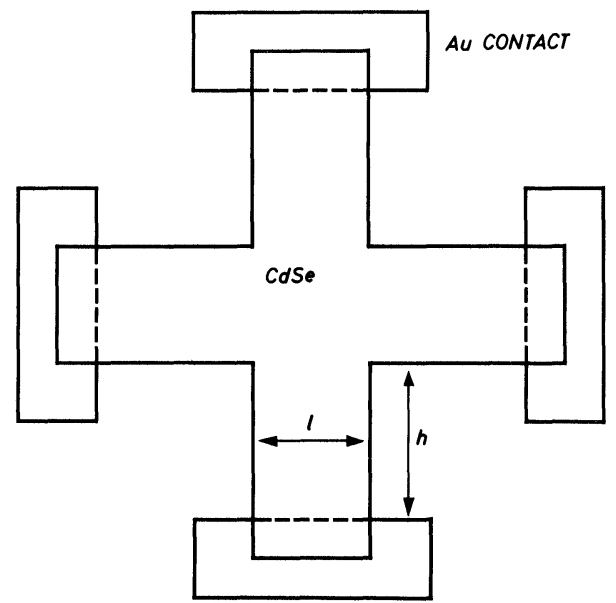

FIGURE 2 Cross shaped sample geometry.

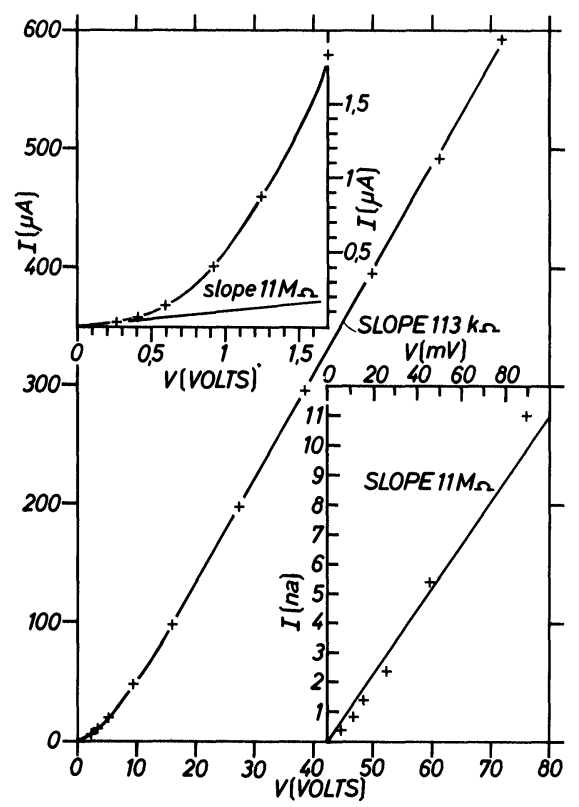

FIGURE 3 I-V characteristics of CdSe sample with underlying $\mathrm{Au}$ contacts.

current plot of a zener diode. Presumably this effect is just avalanche breakdown. The breakdown voltage is 5 volts. On the same sample a Hall mobility of 30 $\mathrm{cm}^{2} / \mathrm{V}$. sec and a carrier concentration of $10^{18} \mathrm{~cm}^{-3}$ was measured. Samples having a higher carrier concentration $\left(>10^{19}\right)$ show no breakdown voltage. Other evidence that the assumption may be correct is that the blocking contact was damaged when a current of $1 \mathrm{~mA}$ was supplied. This is easily explained by the fact that a heat dissipation of $5 \mathrm{~mW}$ then exist 


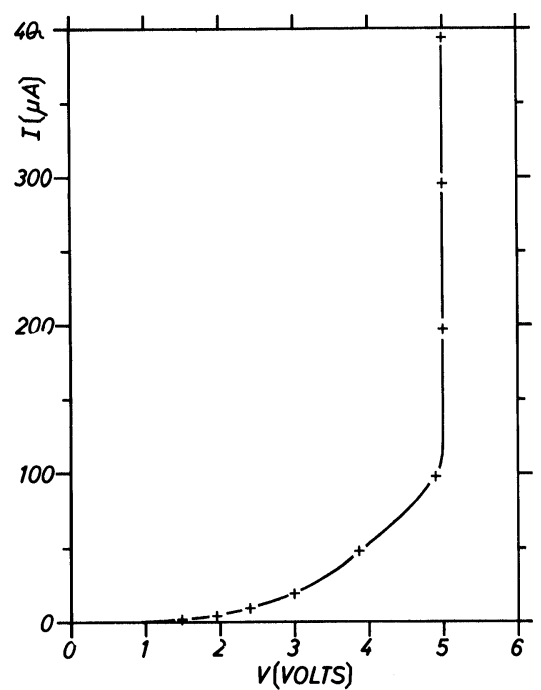

FIGURE 4 Characteristic of reverse biased Au-CdSe diode

in the shallow space charge layer. It is also assumed that the CdSe-metal system may be analysed independently of surface states as has been done with CdS-metal systems ${ }^{3}$.

One concludes that the conditions in a Au-CdS contact are not fulfilled in a Au-CdSe contact; either the first condensed CdSe layers are not sufficiently Cd-rich, or the difference in work functions is too large.

After these experiments, silver conducting ink was used as the contact material. These contacts were perfectly ohmic as can be seen from Figure 5. Even in the nanoampère range the slope of the curve did not vary appreciably. The new value of $230 \mathrm{k} \Omega$ for the semiconductor resistance was caused by a thermal treatment of the sample (baking at $100^{\circ} \mathrm{C}$ for one hour). Good ohmic contacts were also obtained by using evaporated Aluminium.

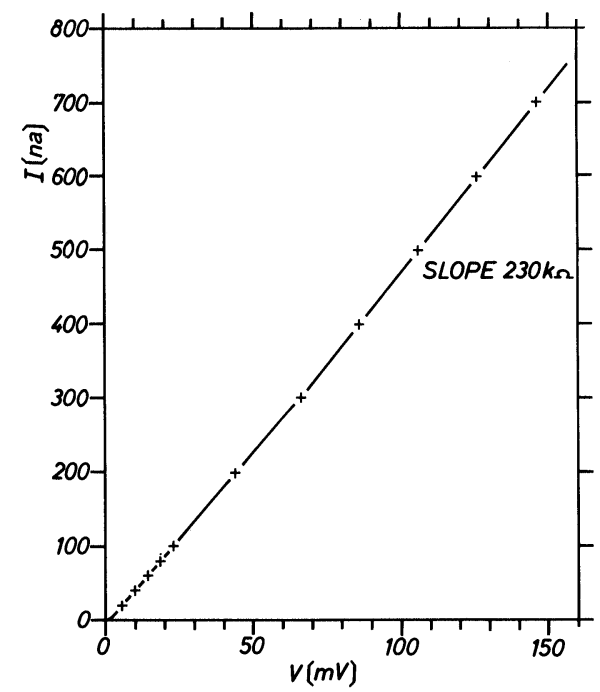

FIGURE 5 Linear I-V characteristic of CdSe sample with silver ink contacts.

The strong dependence of the I-V characteristics on the used metals ( $\mathrm{Au}, \mathrm{Al}$ or silver ink) proves that the surface states do not play an important role, otherwise the junction characteristics would be less dependent on the metal used, as reported by Pfeifer ${ }^{1}$.

\section{REFERENCES}

1. H. Pfeifer; Halbleiterelektronik Wissenschaftliche Taschenbücher (Akademie Verlag, Berlin, 1970), p. 49-58.

2. P. K. Weimer; Thin Film Field Effect Transistors (Prentice Hall, Englewood Cliffs, N.J., 1966).

3. A. C. Tickle; Thin Film Transistors (John Wiley and Sons, New York, 1969) p. 74-78.

4. G. De Mey; 'Influence of sample geometry on Hall mobility measurements' Archiv für Elektronik und Uebertragungstechnik, 27, p. 309-313. (1973). 

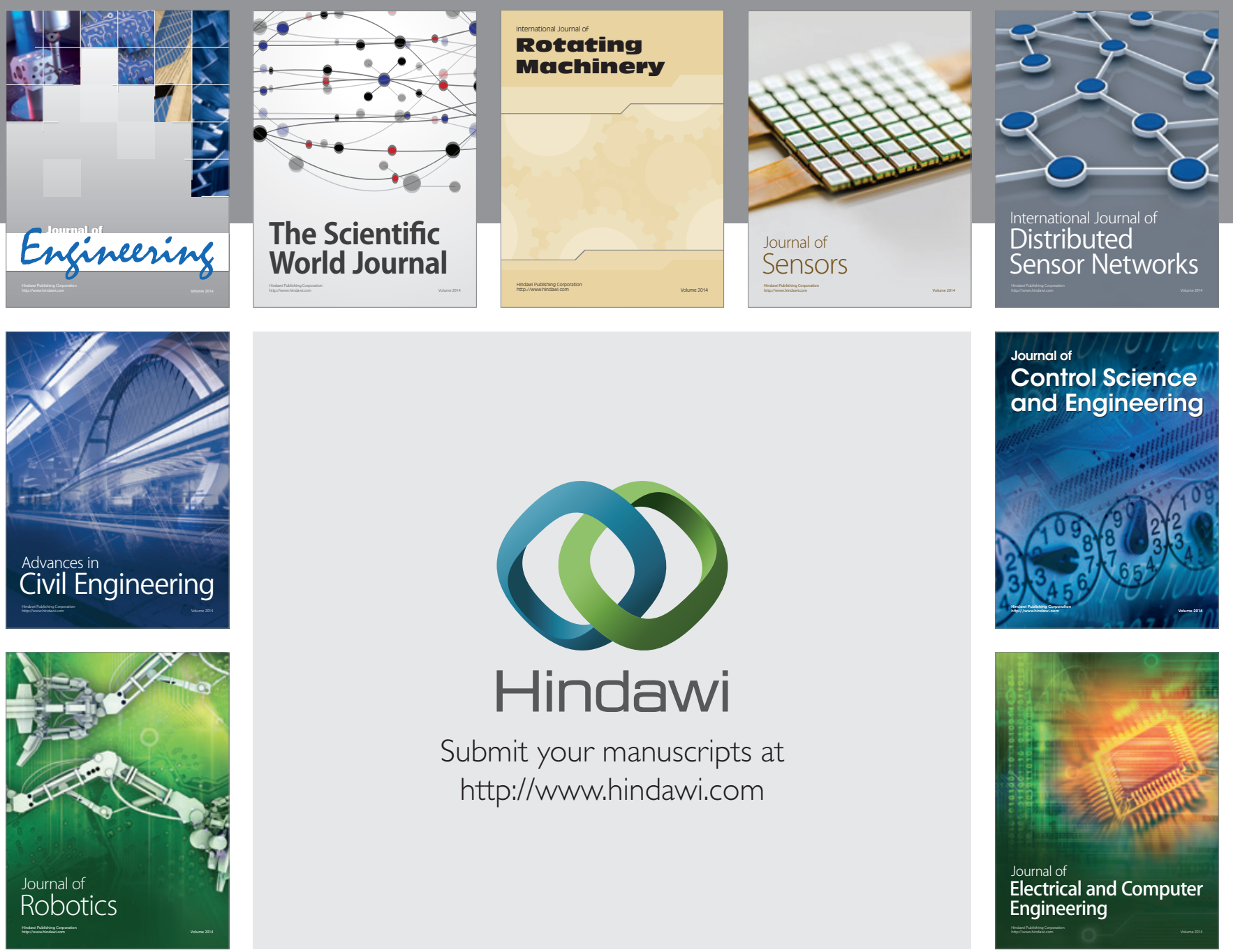

Submit your manuscripts at

http://www.hindawi.com
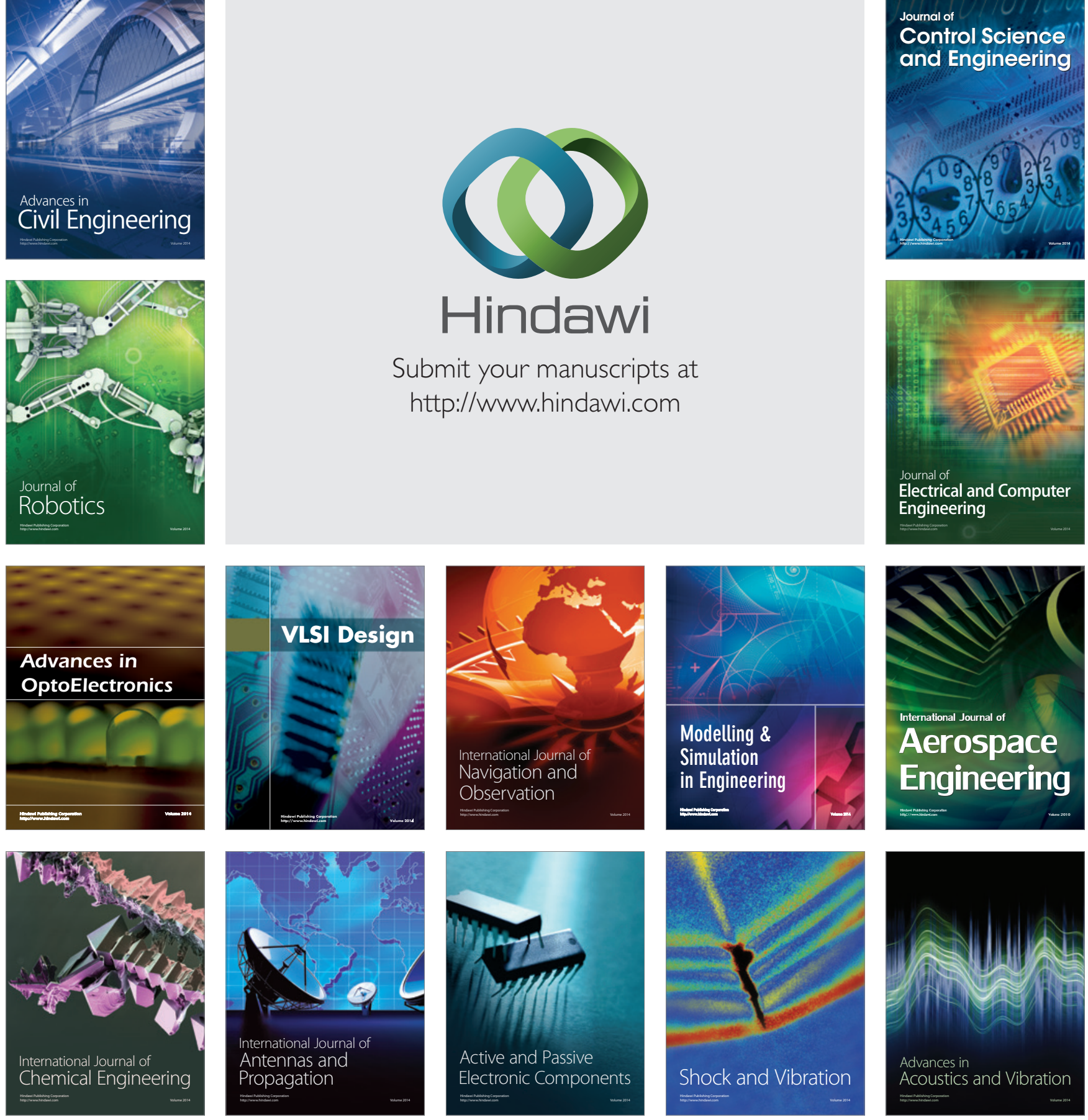